\title{
The Outstanding Effect of Kayak Athletes Psychological Stress Relaxation from Workout for Water Sports Center in Jiangxi Province
}

\author{
Li You, Huang Wenying, Zhang Xiaorong, Qian Fang
}

Sports institute, Jiangxi normal university, Nanchang 330022, China

Keywords: workout; Kayak athletes; Psychological pressure; Attention.

\begin{abstract}
: to understand the pre-match training factors on the influence of stress relaxation and attention athletes, using biofeedback instrument testing, understand the status quo of the athletes' psychological pressure, analyzing the exercise frequency, time, purpose, etc. The water sports center in jiangxi province selected kayak male athletes biofeedback instrument testing, profiling exercise stress relaxation and attention degree of athletes, draw relevant Suggestions: physical exploration, highlighting the pre-match training service; Open sports development, highlight the inclusive of the workout; Successful practice of sports, highlighting the pre-match training of competitive.
\end{abstract}

Since the $1930 \mathrm{~s}$, the psychological pressure of more and more get people's attention. Due to continuous severe social survival environment, increasing employment pressure, high-level event athletes psychological stress is in the peak period of rapid development, when face many pressure for a long time, easy to form psychological confusion, so how to reduce or intervention athletes psychological pressure, maintain their physical and mental health has become a good kayak athletes life in our province is the most sensitive issue. The daily training as a player an important part of life, in promoting athletes plays an important role in mental health [1]. Attention is a kind of indispensable spirit of survival, in terms of greatly affected the athletes in the face of learning, life and work pressure and confused state of mind in the future. So, improving athletes willpower and focus on the future growth of the athletes have a positive role which cannot be ignored. Under the background of new era, the daily training not only to improve the quality of the basic body, at the same time also to be able to affect the athletes' attention, thus effectively improve the comprehensive quality of athletes [2]. The athletes' psychological stress, characterized by mood swings, depression and anxiety symptoms such as representation, seriously affected the athlete's study and life. And daily training can not only make the athletes improve the body quality, more can effectively alleviate the psychological pressure, promote the healthy development of the athlete body and mind [3]. In the new period, to explore the influence degree of the pre-match training for athletes psychological pressure, take more effective measures to improve the workout "decompression" efficiency, has become an important topic in front of us.

\section{The research object and methods}

Water sports center in Jiangxi province were randomly selected excellent 10 kayak male athletes. To measure the height, weight, at the same time, record the age, sex, exercise duration of basic information such as personal.

To collect and analyze related literature mainly includes well-known sports of all kinds of journals, newspapers related theory research and practice situation analysis.

By test data, the quantitative analysis of the effect of athletes psychological barriers and modulation.

Experimental method is based on the experimental results of some intervention controlled irrelevant factors, there are certain experimental condition scientifically operating system, and then observed the experimental conditions change, the phenomenon of accompanied to determine conditions and causal relationship between the phenomenon. Biofeedback instrument in college laboratory facilities, on the basis of process, formulate the corresponding mental relaxation instrument in the process of brain wave amplitude and numerical tests to reflect the effects of 
pre-match training for athletes.

\section{The establishment of the experimental model}

Table 1 the subject basic table

\begin{tabular}{cc}
\hline & Male subjects \\
Class don't & Numerical range \\
\hline Age(years) & $24.00 \pm 0.76$ \\
height (m) & $1.85 \pm 0.04$ \\
weight (kg) & $74.28 \pm 5.50$ \\
Fixed number of year(years) & $5.88 \pm 2.36$ \\
\hline
\end{tabular}

Experiment to the screening of 10 excellent athletes, divided into exercise ( $\mathrm{M}$ group) and the control group (F group), compared before and after contrast. Its age, height, weight, and movement of basic situation, as shown in table 1. Objects are no smoking history, before the trial within $48 \mathrm{~h}$ of insomnia, alcohol or heavy load training, competition, etc., and why not contain suitable for engaged in experimental related matters, have explained many times experiments process at the same time.

\section{The research content}

Study the influence of the pre-match training for athletes psychological pressure, the first step to the analysis of the effect of exercise frequency of psychological pressure if there is a difference, if there is any difference is further analyzed the influence of other factors. Results suggest that are shown in table 2. Comparing the statistical results, and further analyzes that exercise three times a week or more and 1 time/week, occasional exercise, and no exercise groups have significant differences, 2 times/week with occasional exercise and no exercise groups have significant differences. That is more than 3 times per week or exercise can reduce pressure on the players most, 2 times/week of exercise to slow down the psychological pressure is better also.

Table 2 different exercise frequency group of athletes sports strength comparison

\begin{tabular}{cclcccc}
\hline The frequency & 3 times a week or more & 2 times a week & 1 times a week & Occasional exercise & No exercise \\
\hline $\mathrm{N}=10$ people & 5 & 2 & 2 & 1 & 0 & \\
\hline
\end{tabular}

Biofeedback (Biofeedback, BF) refers to the application of modern science and technology, people don't realize normal body biological signals, such as electrical, electrical, skin temperature, heart rate, blood pressure, etc. Into can be aware of the signals, such as visual, auditory signals, according to these signals, so that the patients learn within a certain range through conscious activity regulation of internal organs, to correct the deviation from the normal range of visceral activities treatment and training method [4].Biofeedback Technology (Biofeedback Technology, BT) is through the instrument and the physiological function of human body to make careful measurement and dynamic display, make the physiological parameter information, and timely feedback to the human body. Biofeedback is of the essence of the body function of the human body not easily perceived will signal into can be human visual perception of visual, auditory signals, in order to help the participants to conscious control, to achieve the purpose of the treatment of certain diseases, and biofeedback technologies is the realization of such a signal conversion ways or tools. Yi-jie geng investigate according to Miller had published in Science of the study of the internal organs and glands reaction "proved that the autonomic nervous system control of the internal organs and glands can also establish operant conditioning, and for the biological feedback treatment of psychosomatic disease laid the theoretical basis of physiology. People later combined with Jacobson and Schultz put forward in the $1930 \mathrm{~s}$ of the relaxation therapy and self-training method, gradually created the biofeedback relaxation training treatment [5].Then expanding biofeedback therapy of relaxation training in the field of clinical medicine application, widely used in high blood pressure, coronary heart disease, arrhythmia, diabetes, chronic pelvic inflammatory disease, chronic diseases such as stress urinary incontinence and postoperative rehabilitation of cerebral apoplexy, 
chemotherapy and HRV and so on; And gradually expand to the field of psychology, applied to the anxiety, depression, psychological disorders such as autism, and the pre-match psychological regulation in the areas of research and practice exploration, and achieved fruitful results.

To select indicators for EEG biofeedback relax (alpha), concentration (SMR) wave effect indexes such as power spectrum.

Using biofeedback instrument and the changes of brain wave amplitude of mature testing sports group, concrete manifestation for relaxation (alpha), concentration (SMR) wave. Specific means is: athletes use instrument testing under the quiet state, enter the management interface of biological feedback treatment system pressure, through the selection of the appropriate section stress management, stress management therapy section, stress management relax recovery section in the three sections of the testing subjects. The test time about 20 minutes each.

The EEG data into excel spreadsheet, choose relaxation (alpha), concentration (SMR) wave value, average after collected from each group data, to observe their relaxation, concentration, the data in table 3.

Table 3 biofeedback instrument in M group and group F alpha, SMR wave amplitude numerical comparison

\begin{tabular}{lcccc}
\hline Indicators & The number of & \multicolumn{1}{c}{ M group } & F group & \multicolumn{2}{c}{ The numerical comparison } \\
\hline Alpha (A) & Five people & $47.216 / 64.106 / 69.03$ & $72.744 / 106.08 / 126.456$ & $\mathrm{~F}>\mathrm{M}$ \\
Alpha (B) & Five people $34.822 / 28.164 / 58.872$ & $39.908 / 23.328 / 74.678$ & $\mathrm{~F}>\mathrm{M}$ \\
SMR wave (A) Five people 36.512/50.15/55.698 & $56.716 / 84.582 / 104.21$ & $\mathrm{~F}>\mathrm{M}$ \\
SMR wave (B) Five people 27.696/20.706/48.506 & $30.158 / 16.762 / 59.5$ & $\mathrm{~F}>\mathrm{M}$ \\
\hline
\end{tabular}

Biofeedback test data is mainly analyzed the athlete exercise group and control group in the left brain and right brain (B) (A), it is because this study is to test the pre-match training's influence on the stress relaxation and attention, and (B) in the brain's right hemisphere is mainly responsible for thinking in images, image in imagination thinking is given priority to; The left brain is mainly responsible for (A) logic and computational thinking. So we mainly about sports and non-sports group of athletes brain biofeedback test data for analysis.

By table 3 shows, Alpha waves mainly reflect the subjects relaxation condition, compared two groups of amplitude values, says relaxation of Alpha wave comparison shows significant difference, in our tests is less than the group F M group. The reasons for this situation may be due to the athlete study and living environment is different, have different pressures. Said the attention of SMR wave numerical performance is also a common slants big, the result is smaller than that of group $\mathrm{F} \mathrm{M}$ group. Alpha wave's main purpose is to make the participants through training the Alpha wave increase in mind, so as to improve the attention of the participants and the status of the memory.

\section{The problems existing in the research}

The purpose of this research basic level, but also shortcomings, mainly:

(1) to understand the project groups of athletes is not comprehensive enough, can not fully reflect the athletes' psychological quality characteristics.

(2) the sample size is too small, does not represent the general characteristics of each group athletes.

(3) the psychological intervention time is too short. Due to time constraints, we are subjects of athletes psychological intervention is only 20 minutes, enough skills in psychological skills, psychological training effect is not fully reflected.

(4) lack of pretest and posttest. During the period of psychological training, water sports center in Jiangxi province 10 outstanding men kayak athletes, not a pretest and posttest, can only reflect the subjects close to a period of time or is the psychological characteristics of stage now, not system test out of biofeedback instrument for psychological change characteristics of the subjects. Mainly due to the school time is limited, result in practice is difficult to realize more reasonable design scheme. 


\section{Studies suggestions}

Having the right perception and attribution is an important premise of alleviate psychological pressure. Pre-competition training, can make the athlete to set up the correct outlook on life and world view, make the athletes in order to look more relaxed state of mind, learn to correct attribution [6]. For example, some athletes with poor grades but his athletic ability is stronger, in sports can meet the "self-actualization" and "respect" senior psychological need. In moral education activities, can make the athlete to form scientific values, could actually look at problems from various perspectives more reasonable.

Emotions are athletes for their success in the possibility and necessity of evaluation and experience, Ellis's central "optimal arousal level" theory is that by workout activity, athletes can successfully implement empathy, an athlete of the central nervous get enjoyable experience, so as to eliminate the emotional exhaustion, generates positive emotions. Pre-competition training, can make the athletes get effectively release the bad mood, make the player more rapid, effective empathy, forget the unpleasant emotional experience, abandon the success and failure, enterprising and frustration, joy and pain, selflessly into free release and relief space [7].

Looked from the expectation of incentive theory, the size of the American psychologist FeiLuM think people incentive is affected by the titer and expected probability, expectancy theory is a kind of "procedural" incentive, starting from the basic characteristics of the people, to the person's motivation to act as the psychological process of process of deduction and overview of the formulation, illustrates the role of incentives general regularity relationship between size and titer and expectations [8].In the days before the training, have a fixed role, and this role will be different from life, make the athletes to establish an appropriate effect value; At the same time, to encourage, between the athlete through peer recognition, set up to overcome all the difficulties of courage and confidence, the formation of the expectations of a higher probability, eventually improve the titer of the athletes, the athletes keep up positive, healthy mental state, make the athlete to the life full of expectation and agency again.

American psychologist fadden berg had pointed out that "the best way to alleviate the psychological pressure is communicating with people, in the open communication enhance the individual's self-efficacy". In the field of sports, many sports belong to the collective activity, athletes in sports activities of a series of "informal group" in the "informal group", athletes can associate with other athletes, more make the athlete society cooperation, communication, the athletes to certain common goals to strive, to form the collective centripetal force [9].Pre-competition training, make the athletes to form a bright and cheerful disposition, optimistic attitude toward life, athletes and unhappy, forget the short trouble eliminate loneliness.

\section{Acknowledgements}

The paper is sponsored by the Postgraduate Innovation Foundation Project of Jiangxi Normal University under Project No. YC 2016-S137 and Humanities and Social Sciences Project of Jiangxi Province under Project No. 16 TY07.

\section{References}

[1] Cai Li. Athletes amateur training behavior prediction: based on the research of psychological control [J]. Journal of east China normal university, 2007, (5): 9-10.

[2] Zhang Xiyou. Under the new situation of the need for strengthened workout to explore [J]. China's school education, 2012, (7): 32-35. Not much

[3]. The psychological effects of physical activity and exercise [J]. Journal of sport science. 1997, (3): 75-81.

[4] Zhang Sufan, bitsh Zhou Xiesheng. Biofeedback [M]. Beijing: Beijing science and technology club, 1987 9-50. 
[5] Yuan Xiao jiao, Zhang Yubin, exhibitions, etc. Test anxiety under stress situation biofeedback intervention effectiveness study [J]. Chinese journal of health psychology, 2008, (2), 160-162.

[6] liu jie. Athletes pressure study [J]. Journal of institutions of higher learning in shanxi society proceedings, 2004:80-83.

[7] Phi11ip, shilin. Stress and health [M]. Beijing: China light industry press, 2000:217-227.

Clear [8] season, li Lin, xiao-zan wang. The effect of physical exercise on mental health [J]. Journal of shandong sports institute, 1998. (01): 38 and 43.

[9] YanHengChan Ding Xueqin, Chen Yongjia. Biofeedback technology in the application of athletes psychological training process [J]. Journal of physical education and science, 2000, (3): 44-47. 